\title{
Design and Application of Multi-axis Automatic Control System Based on LabVIEW
}

\author{
Han Ming \\ Tianjin Polytechnic University, \\ TJPU, \\ Tianjin, China \\ hanming.tjpu@163.com
}

\author{
Miao Changyun \\ Tianjin Polytechnic University, \\ TJPU, \\ Tianjin, China \\ miaochangyun@tjpu.edu.cn
}

\author{
Zhu Guangyu \\ Tianjin University of Science \& \\ Technology, TUST, \\ Tianjin, China \\ zhuguangyu312@163.com
}

\begin{abstract}
In the thesis, "Personal Computer(PC) + Motion Control Card" program is adopted to design multi-axis automatic control system and presents the method to control the interface and procedures in LabVIEW through ActiveX technology. The use of servo motors with feedback function could achieve closed-loop control and improve accuracy of the system. This system not only has intuitive interface and simple operation but also has high accuracy and superior performance.
\end{abstract}

Keywords-Multi-Axis Automatic; Motion Control Card; Servo Motor; LabVIEW

\section{INTRODUCTION}

Taking mechanical movement as a main way, automatic control could control the motor quickly and accurately to make sure the moving parts or subsystems complete the corresponding movement in accordance with the operating parameters and the expected track. The variables conclude the motor's position, velocity, acceleration, torque and other parameters.

The automation control system develops from analog to digital, DC to AC, open loop to closed-loop. Every process promotes the development of automatic control systems to a large extent. Closed-loop control ensures the control system is stable, reliable and operates in high-precision.

At present, single-axis control is difficult to meet the practical requirements. Multi-axis control is required in the production of a variety of complex tracks. ${ }^{[1]}$ It is the future development trend for controlling operation quickly, accurately through motion control card and the PC interface to control multi-axis motor movement.

\section{SYSTEM DESIGN}

This system is based on "Personal Computer + Motion Control Card" ,it aims to design a control system of multiaxis motion. The system consists of the PC, motion control card, servo motor drives, servo motors and mechanical actuators. Automatic control is the mechanical motion intended to transform target into the expected result. The objectives of automatic control system conclude position, velocity, acceleration, torque or moment. The system aims at the control of the motor position, velocity and acceleration. Its composition is shown in Figure 1.

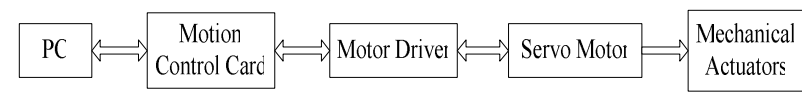

Figure 1. Diagram of automatic control system

Motion control card embeds in the industrial machine and it constitutes the multi-axis automatic control system based on "Personal Computer + Motion Control Card " model. ${ }^{[2,3]}$ The PC is connected through the PCI bus and motion control card which send movement signal to the motor drive to drive servo motor and control the movement of mechanical actuator. Motion control card can control motor's position, interpolation drive, acceleration and deceleration by outputting high-frequency pulse to control the movement of the servo motor and motor speed. The number of pulses controls the motor corner and the pulse frequency rate controls acceleration. After receiving signals from motion control cards, motion drive will send motor signals and control signals to servo motor. Servo motor rotates and controls the motor speed, angle, acceleration and deceleration and direction of movement. The rotation of servo motor drives mechanical actuator movement to achieve movement control.

Motion control card of the system is the ACR1505 from Parker Hannifin Corporation. Compared with other products, it has high performance and speed, control precision and convenient operation system. It uses 32-byte floating-point DSP with 120MFOPS processing capability, including four $30 \mathrm{MHz}$ encoder input. It can control multi-axis servo motor or stepper motor control via the connection between PCI bus and the PC. Servo motors with feedback function is selected. The system is closed-loop control, so it can feedback the object's location, speed and other information immediately. It can improve the accuracy of the control system and the operation is more simple and intuitive. 


\section{SELECTION AND APPLICATION OF LABVIEW}

LabVIEW is raised compared with the traditional material instruments. That means the imaginary instrument is built through constructing electronic equipment. A virtual instrument system is usually constituted with powerful application software which supported by an industrystandard computer or workstation and low-cost hardware (such as plug-in boards) and driver software components. ${ }^{[4]}$ All these together can fulfill the functions of traditional instruments.

The virtual instrument can take advantage of the highperformance modular hardware combined with efficient and flexible software to complete a variety of tests, measurements and automatic control operations. Its function and role are almost the same with those traditional material instruments, but it can save cost significantly and free from the inconvenience and fragility of the traditional instrument. With virtual instruments, users can set up measurements and control system according to their needs instead of limited by fixed function which decided entirely by the manufacturers.

The most commonly used virtual software is LabVIEW programming software. Its characteristics can provide users with a graphical programming environment, so it is an ideal tool to create virtual instrumentation systems. Users can use LabVIEW to create a graphical interactive interface on computer and design a virtual instrument after their own requirements. Through this graphical interface, users can design operation instrument, control hardware, collect data, analyze and display operation results.

In multi-axis automatic control system LabVIEW need the ActiveX plug-in to call the motion control card. Through ActiveX automation LabVIEW can be both a client and a server. As a client, LabVIEW can call objects in ActiveX automation server and access its properties and methods which can be used in programming. This paper is based on calling motion control card in LabVIEW platform by using ActiveX technology to achieve further programming. It can control the position, speed, acceleration and other parameters of the multi-axis automatic control. It can speed up the development speed of multi-axis automatic control system and shorten the development cycle considerably.
The ACR1505 motion control card provides an ActiveX automation server called "ComACRsrvr" which contains four ActiveX objects: the status, control, terminal and utility. These ActiveX objects provide a wide range of properties and methods of operation of the motion control card. Among them, the status provides status information and relative properties and methods; control provides properties and methods can achieve the automatic control parameter data (such as position, velocity, etc.) and it makes implement more simple to control. Through calling these ActiveX objects and accessing their properties and methods, control and operation of motion control card can be achieved.

\section{IMPLEMENTATION OF PROGRAM}

After ActiveX automation is required in application of LabVIEW program in multi-axis automatic control system. With LabVIEW as an Automation client, the motion control card functions as an automation server, LabVIEW can call objects in motion control card and access its properties and methods. LabVIEW provides a lot of manipulation functions in Connectivity-an ActiveX function panel. ${ }^{[5]}$ The main function Automation Open is used to open the ActiveX object to obtain the Reference; the Property the Node is used to read or set the properties of the ActiveX object; the Invoke Node is used to call the ActiveX object.

Firstly copy ComACRsrvr provided by ACR1505 movement control card to the root directory of LabVIEW. Then place Automation Open function in the LabVIEW block diagram and open the reference to the ActiveX automation. And create a control connected with Automation Refnum in the upper left angle so as to select and call of the ActiveX object. Place one or more methods (or properties) of the node function Invoke the Node (or the Property Nodes) in the block diagram and connect the right end of Automation Open function with Reference input of method nodes. Each node must be sequentially connected end to end to access more than one property and method of an ActiveX object.

The system preliminarily designs the $\mathrm{X}, \mathrm{Y}, \mathrm{Z}$ axes of three-dimensional process control. The front panel interface of LabVIEW is shown in Figure 2.

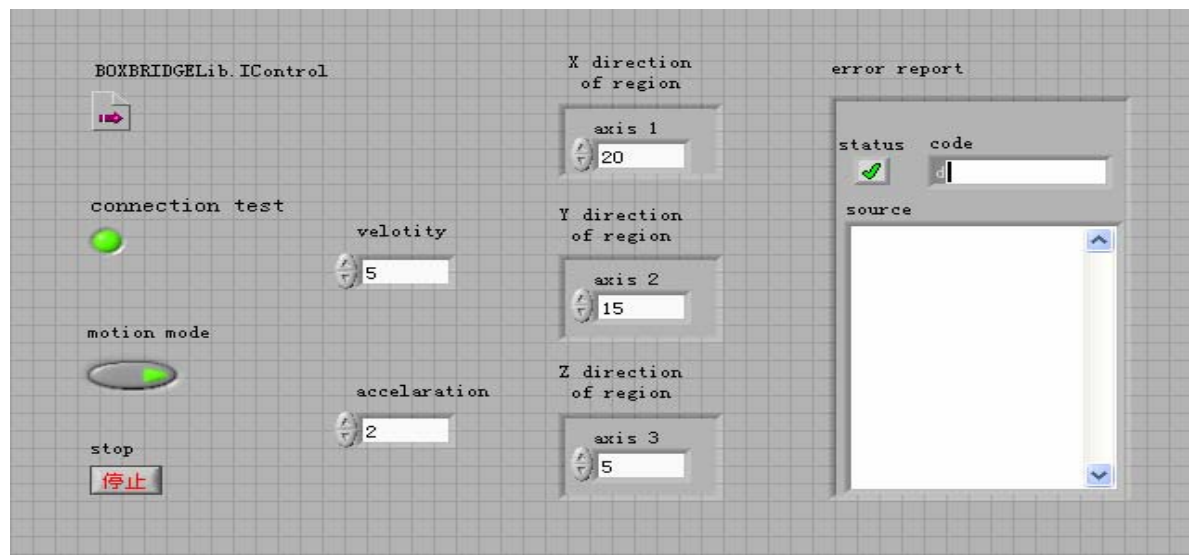

Figure 2. The front panel interface of LabVIEW 
The front panel of LabVIEW includes connection, speed, acceleration, and the parameters of movement of each axis which control multi-axis automatic control system. Whether test light is on or not indicates whether the LabVIEW is properly connected to ActiveX object. Movement modes can be chosen as absolute motion or relative motion to control different objects in different conditions. If any errors occur during system operation, it can be reviewed in the error report section to modify.

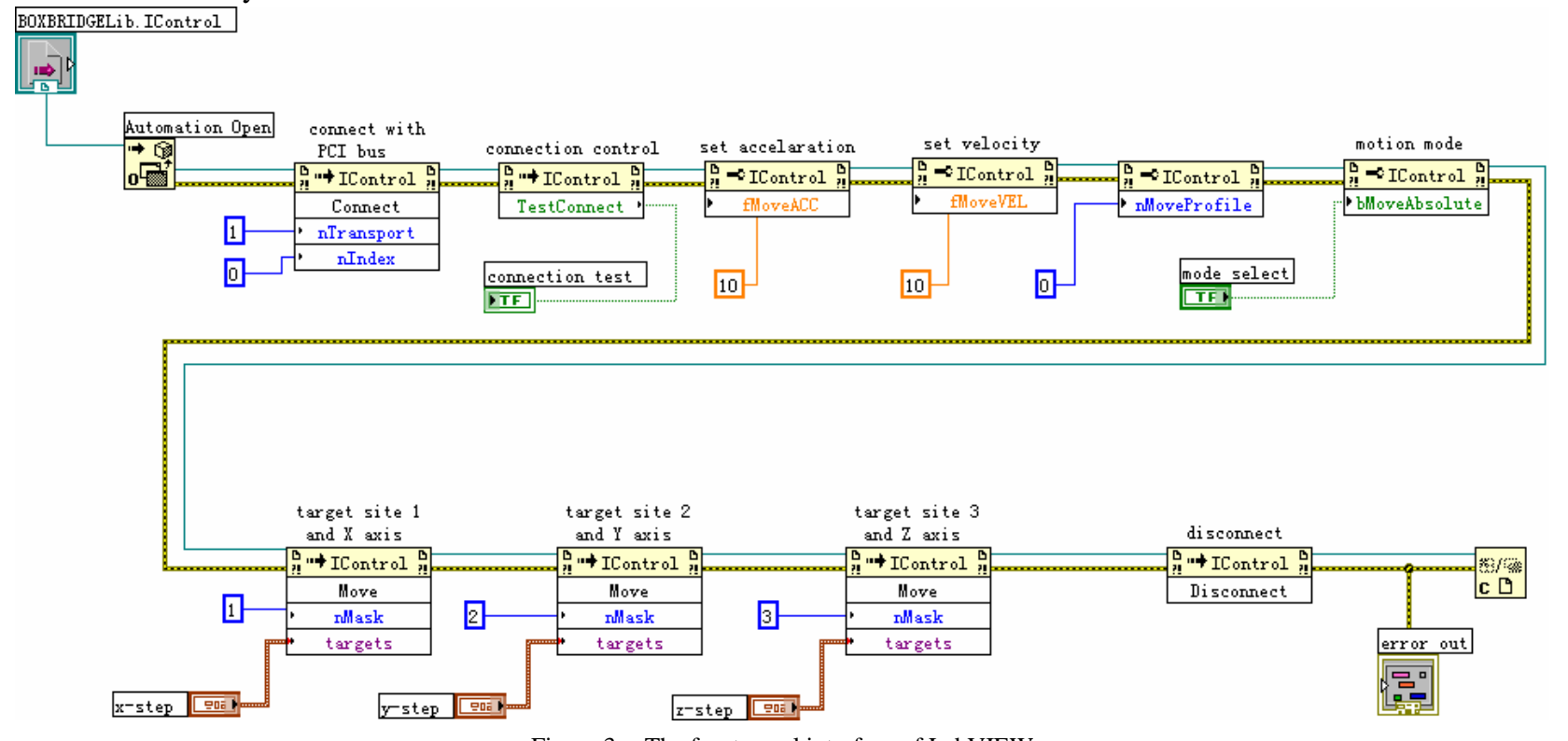

The diagram of multi-axis automatic control is shown in Fig. 3 which fMoveVE, fMoveACC, bMoveAbsolute are used to set the system running speed, acceleration and patterns. Connect is used to set the communication of control card and PC and will be set for the PCI bus, the Move to set the motor position and axis of movement.

Figure 3. The front panel interface of LabVIEW

\section{CONCLUSION}

The thesis aims to design a multi-axis automatic control systems based on "Personal Computer + Motion Control Card "program to research and apply it in the LabVIEW software platform. Compared with other commonly used $\mathrm{VB}, \mathrm{VC}++$, LabVIEW is superior in intuition, simple operation, development potential, low cost and its suitability for system design and development. Application in multiaxis automatic control system can be realized through compiling the interface and control procedures of LabVIEW software and calling the ActiveX and other feature. This system is precise, fast, easy to operate with low cost and ensures instantaneity, reliability and stability in practical applications.

\section{REFERENCES}

[1] Zhaoqing, Wang Qikun. "Design of Multi-axis Motion Controller Based on PC,"Machine Building and Automation, 2005, 117-119.

[2] Wang Shufang. "Multi-Axis Step-motor Control System Based on Virtual Instrument," Microcomputer Information, 2006, 54-57.

[3] Zhou Zhiming. "Step-motor Control System Based on Motion Control Card ," Coal Mine Machinery, 2004, 95-97.

[4] Mao Jiqing. Labview8.2-Weapon for Rapid Construction of Step Motor Control System , Electric Machines \& Control Application, 2009, 30-32.

[5] Zhang Weixing, "Zhao Feng. Research of Motion Control System Based on LabVIEW , ” Industrial Control Computer, 2008, 26-28. 